\title{
Acute administration of ivacaftor to people with cystic fibrosis and a G551D-CFTR mutation reveals smooth muscle abnormalities
}

Ryan J. Adam, ${ }^{1,2}$ Katherine B. Hisert, ${ }^{3}$ Jonathan D. Dodd, ${ }^{4}$ Brenda Grogan, ${ }^{5}$ Janice L. Launspach, ${ }^{2}$ Janel K. Barnes, ${ }^{6}$ Charles G. Gallagher, ${ }^{5}$ Jered P. Sieren, ${ }^{7}$ Thomas J. Gross, ${ }^{2}$ Anthony J. Fischer, ${ }^{8}$ Joseph E. Cavanaugh, ${ }^{6}$ Eric A. Hoffman, ${ }^{1,7}$ Pradeep K. Singh,, ${ }^{3,9}$ Michael J. Welsh, 2,10,11,12 Edward F. McKone, ${ }^{5}$ and David A. Stoltz ${ }^{1,2,10,12}$

'Department of Biomedical Engineering, ${ }^{2}$ Department of Internal Medicine, University of lowa, lowa City, lowa, USA. ${ }^{3}$ Department of Medicine, University of Washington, Seattle, Washington, USA. ${ }^{4}$ Department of Radiology and ${ }^{5}$ National Referral Centre for Adult Cystic Fibrosis, St. Vincent's University Hospital and University College Dublin School of Medicine, Dublin, Ireland. 'Department of Biostatistics, 'Department of Radiology, and ${ }^{8}$ Department of Pediatrics, University of lowa, lowa City, lowa, USA. 9Department of Microbiology, University of Washington, Seattle, Washington, USA. ${ }^{10}$ Department of Molecular Physiology and Biophysics, ${ }^{11}$ Howard Hughes Medical Institute, and ${ }^{12}$ Pappajohn Biomedical Institute, University of lowa, lowa City, lowa, USA.

BACKGROUND. Airflow obstruction is common in cystic fibrosis (CF), yet the underlying pathogenesis remains incompletely understood. People with CF often exhibit airway hyperresponsiveness, CF transmembrane conductance regulator (CFTR) is present in airway smooth muscle (ASM), and ASM from newborn CF pigs has increased contractile tone, suggesting that loss of CFTR causes a primary defect in ASM function. We hypothesized that restoring CFTR activity would decrease smooth muscle tone in people with CF.

METHODS. To increase or potentiate CFTR function, we administered ivacaftor to 12 adults with CF with the C551D-CFTR mutation; ivacaftor stimulates C551D-CFTR function. We studied people before and immediately after initiation of ivacaftor ( 48 hours) to minimize secondary consequences of CFTR restoration. We tested smooth muscle function by investigating spirometry, airway distensibility, and vascular tone.

RESULTS. Ivacaftor rapidly restored CFTR function, indicated by reduced sweat chloride concentration. Airflow obstruction and air trapping also improved. Airway distensibility increased in airways less than $4.5 \mathrm{~mm}$ but not in larger-sized airways. To assess smooth muscle function in a tissue outside the lung, we measured vascular pulse wave velocity (PWV) and augmentation index, which both decreased following CFTR potentiation. Finally, change in distensibility of $<4.5-\mathrm{mm}$ airways correlated with changes in PWV.

CONCLUSIONS. Acute CFTR potentiation provided a unique opportunity to investigate CFTRdependent mechanisms of CF pathogenesis. The rapid effects of ivacaftor on airway distensibility and vascular tone suggest that CFTR dysfunction may directly cause increased smooth muscle tone in people with CF and that ivacaftor may relax smooth muscle.

FUNDING. This work was funded in part from an unrestricted grant from the Vertex InvestigatorInitiated Studies Program.

Conflict of interest: The authors have declared that no conflict of interest exists.

Submitted: December 28, 2015 Accepted: March 1, 2016 Published: April 7, 2016

Reference information: JCI Insight. 2016;1(4):e86183. doi:10.1172/jci.insight.86183.

Authorship note: E.F. McKone and D.A. Stoltz contributed equally to this work. 
Table 1. Pretreatment study participant characteristics

\begin{tabular}{|c|c|}
\hline \multicolumn{2}{|l|}{ Demographics } \\
\hline Number of subjects & 12 \\
\hline Age (yrs) & $31 \pm 9[22-57]$ \\
\hline Male & 3 subjects \\
\hline G551D-CFTR allele & 12 subjects \\
\hline F508del-CFTR allele & 8 subjects \\
\hline BMI $\left(\mathrm{kg} / \mathrm{m}^{2}\right)$ & $21.8 \pm 3.4[17.1-29.7]$ \\
\hline Sweat chloride (mmol/l) & $94 \pm 16[61-110]$ \\
\hline $\mathrm{FEV}_{1}(\%$ predicted) & $64 \pm 23$ [34-101] \\
\hline
\end{tabular}

caused by loss of CFTR. This is a key problem that has limited progress. A variety of approaches to overcome this problem have been used, including primary cultures of cells and cell lines. Animal models have been especially helpful $(1,2)$. However, studies in humans with the disease are ultimately needed for confirmation (3-6). Here, we used the approach of studying people with CF before and after CFTR restoration to better understand the role of CFTR in smooth muscle function and the primary mechanisms of CF airway disease.

Airway smooth muscle (ASM) abnormalities have been described in people with CF, including increased smooth muscle mass, bronchodilator responsiveness, and airway hyperreactivity (7-13). However, whether these findings are due to the primary loss of CFTR in ASM or secondary confounding factors is unknown and has been difficult to study in humans. CFTR is present in ASM cells (7, 14-16), and newborn CF pigs exhibit airflow obstruction and have increased ASM tone/contraction prior to the onset of airway inflammation and mucus accumulation $(4,16,17)$. These findings suggest that CFTR loss may cause a primary defect in ASM function.

In the current study, we used ivacaftor treatment to acutely augment CFTR function in people with $\mathrm{CF}$ in order to investigate whether smooth muscle abnormalities are a primary consequence of CFTR disruption. Ivacaftor enhances CFTR activity in a subset of people with CF who have CFTR gating mutations, including those with G551D-CFTR (18-21). Ivacaftor increases the open-state probability of active or phosphorylated CFTR, a process termed "potentiation" (21). In people with CF and the G551D-CFTR mutation, ivacaftor treatment leads to sustained improvements in lung function (18, 19, 22-25).

We hypothesized that loss of CFTR function in human ASM increases smooth muscle contraction and contributes to CF airway disease. We tested our hypothesis in people with CF and the G551D-CFTR mutation by investigating smooth muscle function before and soon after initiating ivacaftor treatment. We performed spirometry (before and after bronchodilator), computed tomography (CT) scanning (inspiratory and expiratory chest images) to assess airway distensibility, and noninvasive measures of vascular smooth muscle tone. To minimize secondary consequences of restoring CFTR function, we limited the study duration to 48 hours of ivacaftor treatment, as ivacaftor is estimated to reach near steady state concentration in 2-3 days (26). By using ivacaftor as a tool to restore CFTR function, our goal was to better understand the primary mechanisms of CF airway disease and the role of CFTR in smooth muscle function.

\section{Results}

\section{Ivacaftor rapidly improves CFTR function}

Table 1 summarizes pretreatment subject demographics. Twelve subjects participated in this study. All subjects had at least 1 G551D-CFTR allele. Eight subjects were compound heterozygotic for F508del-CFTR, and in the remaining 4 subjects, the second CFTR mutant alleles were: $3659 \mathrm{delC}, P 67 \mathrm{~L}, \mathrm{G551D}$, and $R 117 \mathrm{H}$. Nine of the study subjects were on an inhaled short-acting bronchodilator, and 8 were on an inhaled long-acting bronchodilator prior to the study. Only 1 subject was not using any inhaled bronchodilator therapy. Before ivacaftor treatment, subjects underwent spirometry, whole-lung inspiratory and expiratory chest CT scanning, and noninvasive measures of vascular tone. Forty-eight hours after starting ivacaftor therapy, subjects returned to the clinic and repeated the examinations that had been completed on day 0 . We used sweat chloride concentrations to assay CFTR function. Forty-eight hours after starting treatment, sweat chloride 

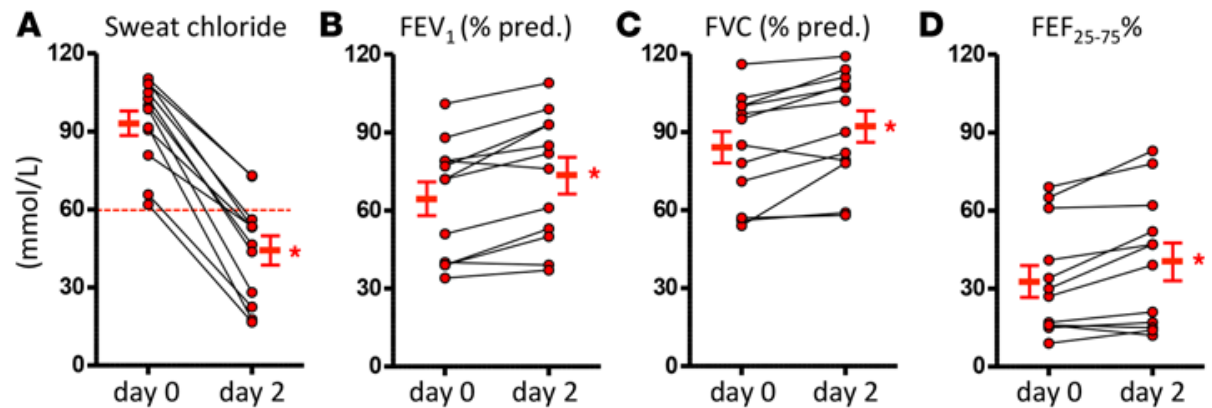

Figure 1. Ivacaftor treatment rapidly improves sweat chloride concentration and airflow obstruction. (A) Sweat chloride concentration (mmol/I). Dashed line indicates diagnostic threshold for cystic fibrosis (60 mmol/I). (B) Forced expiratory volume in 1 second, percent predicted (FEV 1 [\% pred.]). (C) Forced vital capacity, percent predicted [FVC (\% pred.)]. (D) Forced expiratory flow rate $25 \%-75 \%\left(\mathrm{FEF}_{25 \%-75 \%}\right)$. Studies were performed day 0 (before ivacaftor) and following 2 days of ivacaftor treatment. In individual panels, each symbol represents a different subject. Connected symbols represent individual subjects before and after ivacaftor. Horizontal bars represent mean \pm SEM, and a paired $t$ test was performed. ${ }^{*} P \leq 0.01$.

concentration decreased from an average of $94 \pm 5 \mathrm{mmol} / 1$ to $45 \pm 6 \mathrm{mmol} / 1$ (mean $\pm \mathrm{SEM} ; P<0.0001$, Figure 1A). These data indicate that ivacaftor acts very quickly and CFTR potentiation can be observed within 48 hours of beginning treatment.

\section{Ivacaftor quickly improves airflow obstruction}

The average pretreatment forced expiratory volume for 1 second $\left(\mathrm{FEV}_{1}\right)$ (percentage predicted) was $64 \%$ $\pm 7 \%$ (mean \pm SEM). Two days of ivacaftor treatment reduced airflow obstruction, leading to increases in absolute values of $\mathrm{FEV}_{1}(2.1 \pm 0.31$ vs. $2.4 \pm 0.31, P<0.05)$, forced vital capacity (FVC) ( $3.3 \pm 0.31$ vs. 3.6 $\pm 0.31, P<0.05)$, and forced expiratory flow rate $25 \%-75 \%\left(\mathrm{FEF}_{25 \%-75 \%}\right)(33.3 \% \pm 6.1 \%$ vs. $40.6 \% \pm 7.3 \%$, $P<0.05$ ) and the corresponding average percentage of predicted values (Figure 1, B-D).

\section{Ivacaftor reduces air trapping}

Air trapping is caused by airway narrowing or obstruction $(17,27-30)$ and is one of the earliest radiographic findings in people and pigs with $\mathrm{CF}(17,27,28)$. We used $\mathrm{CT}$ imaging to assess air trapping, which we defined as the percentage of lung CT scan voxels less than -856 Hounsfield units (HU) on expiratory CT scans. Figure 2 shows $\mathrm{CT}$ scan images from a study subject, before and after ivacaftor treatment, demonstrating an improvement in air trapping. On a whole-lung basis, there was a trend for less air trapping after 2 days of ivacaftor treatment, although the average change failed to reach the significance level of $P<0.05$ ( $P=0.052$ for Wilcoxon matched pairs signed-rank test and $P=0.069$ for paired $t$ test; Figure 2 and Figure $3 \mathrm{~A})$. We also assessed regional changes in air trapping. Before treatment, air trapping tended to be greatest in the upper third of the lung, consistent with the known predilection for $\mathrm{CF}$ disease in the upper lung regions. Ivacaftor reduced air trapping in all 3 regions; however, only for the lower third was this reduction significant statistically (Figure 3B). Baseline air trapping values (\% CT scan voxels $<-856$ $\mathrm{HU}$ on expiratory scans) strongly correlated with the baseline residual volume (RV)/total lung capacity (TLC) lung volume ratio, another measure of air trapping (31), suggesting that we were measuring regions of air trapping and not regions of hypoperfusion $(P<0.01, \mathrm{r}=0.82$; Figure $3 \mathrm{C})$. Despite improvements in

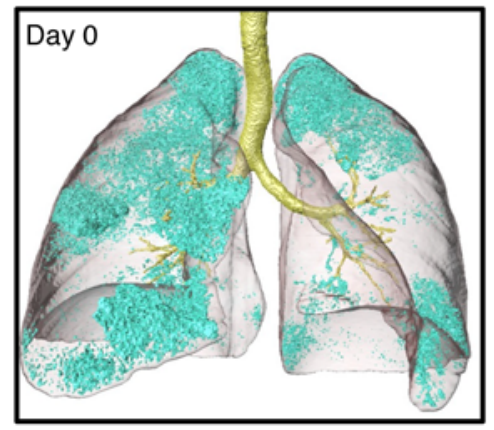

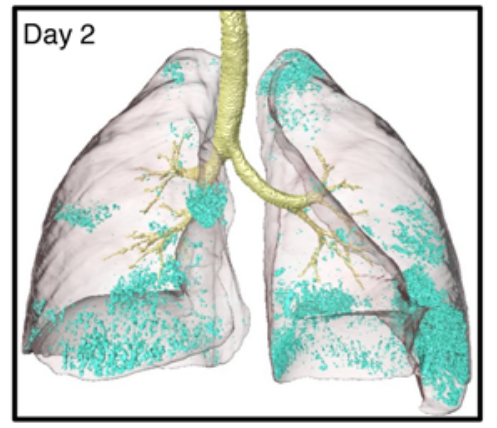

Figure 2. Ivacaftor improves CT-based air-trapping assessment. CT-derived 3-dimensional rendering of the lung and airways from a study participant before (day 0 ) and after ivacaftor treatment (day 2). Regions of air trapping (Hounsfield units $[\mathrm{HU}]<-856)$ are highlighted in light blue. 


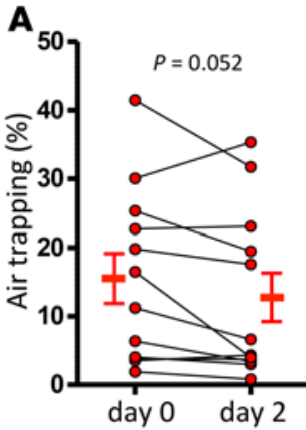

B

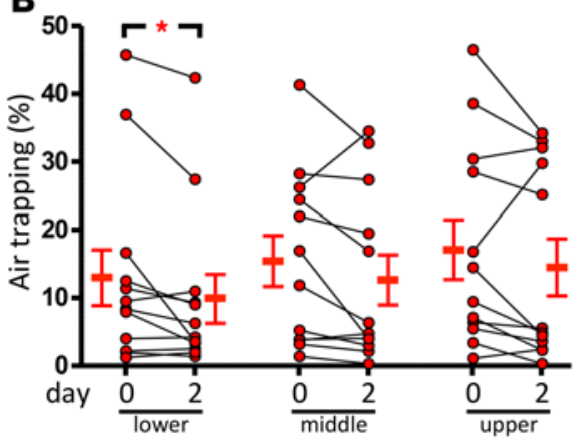

C

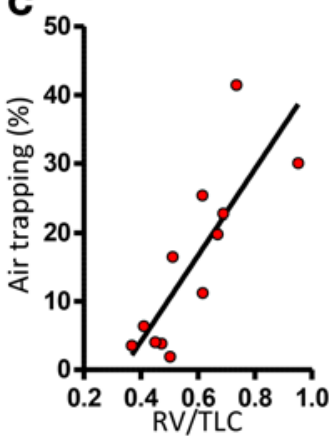

Figure 3. Ivacaftor rapidly improves air trapping. Studies were performed on day 0 (before ivacaftor) and following 2 days of ivacaftor treatment. (A) Total lung air trapping. Air trapping was defined as the percentage of voxels below -856 Hounsfield units (HU) on the RV (expiratory) CT scan. (B) Regional lung air trapping. (C) Correlation between air trapping (day 0 ) defined by the percentage of total lung voxels below $-856 \mathrm{HU}$ vs. air trapping defined by the RV/ TLC ratio. The RV (expiratory) and TLC (inspiratory) lung volumes were determined from CT datasets (day 0). In individual panels, each symbol represents a different subject. In $\mathbf{A}$ and $\mathbf{B}$, connected symbols represent individual subjects before and after ivacaftor. $n=12$ subjects. Horizontal bars represent mean \pm SEM. A Wilcoxon matched pairs signed-rank test (A) or a paired $t$ test (B) was performed. A Pearson's correlation coefficient ( $r$ ) was determined for data in $\mathbf{C} .{ }^{*} P<0.05$. RV, residual volume; TLC, total lung capacity.

air trapping, the average inspiratory and expiratory lung volumes were not statistically different before and after ivacaftor treatment (Figure 4, A and B).

Seven of 12 subjects had improvements in both total lung air trapping and $\mathrm{FEV}_{1}$ (\% predicted). These improvements were significantly correlated; individuals who improved the most in FEV (\% predicted) also improved the most in air trapping after only 2 days of CFTR restoration $(P=0.02, \mathrm{r}=-0.67$; Figure $4 \mathrm{C})$. We also observed a strong correlation between spirometrically obtained FVC and CT-derived vital capacity, defined as the difference between lung volumes obtained from the TLC (inspiratory) and RV (expiratory) CT scans $(P<0.0001, r=0.91 ;$ Figure 4D). The strong correlation between spirometry and CT data suggest well-executed and repeated subject performance for both spirometry and CT scan breathing maneuvers.

\section{Restoring CFTR activity reduces $\beta$-agonist-induced bronchodilation, increases airway} distensibility, and decreases vascular tone

We hypothesized that the rapid reversal of airflow obstruction following CFTR potentiation was, in part, due to an effect on ASM. We tested this hypothesis by investigating measures of smooth muscle tone before and after restoration of CFTR function. These studies included the bronchodilator response, airway distensibility measurements (from CT scans), and vascular tone.

Bronchodilator response. Salbutamol (albuterol) is a short-acting $\beta_{2}$ agonist that acts as an ASM relaxant and improves airflow $\left(\mathrm{FEV}_{1}\right)$ in individuals with airway obstruction caused by ASM contraction.
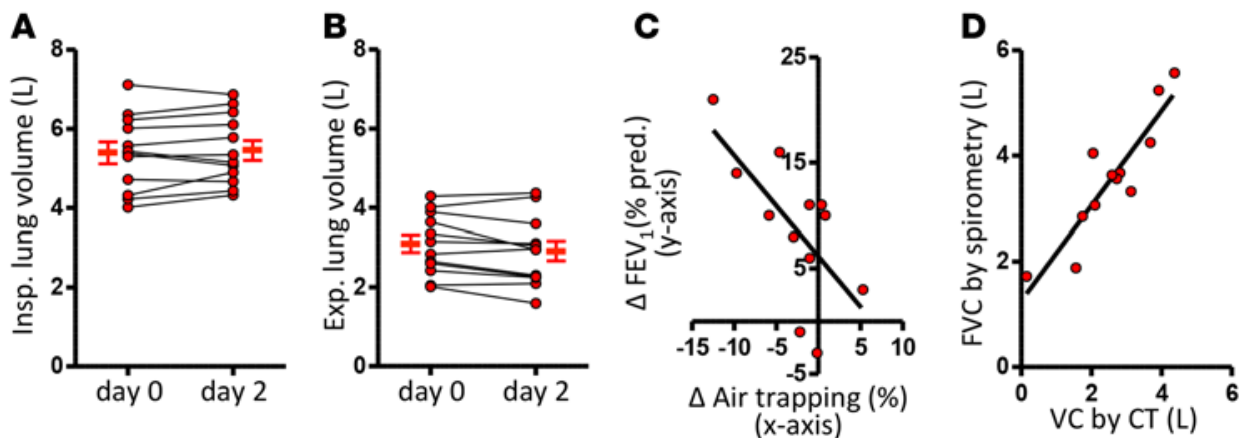

Figure 4. Less air trapping correlates with improved spirometry. (A) Total inspiratory lung volume and (B) Total expiratory lung volume from CT datasets on day 0 (before ivacaftor) and following 2 days of ivacaftor treatment (day 2). (C) Correlation between changes in total lung air trapping (percentage of lung voxels below -856 Hounsfield units [HU]) and changes in forced expiratory volume in 1 second (FEV) (\% predicted) before and after ivacaftor. (D) Forced vital capacity (FVC, liters) from spirometry and vital capacity (VC, liters) from CT-based quantification after ivacaftor. For individual panels, each symbol represents a different subject. In $\mathbf{A}$ and $\mathbf{B}$, connected symbols represent individual subjects before and after ivacaftor. Horizontal bars represent mean $\pm S E M$. A paired $t$ test was performed for data in $\mathbf{A}$ and $\mathbf{B}$. A Pearson's correlation coefficient $(r)$ was determined for data in $\mathbf{C}$ and $\mathbf{D}$. ${ }^{*} P<0.05$. 

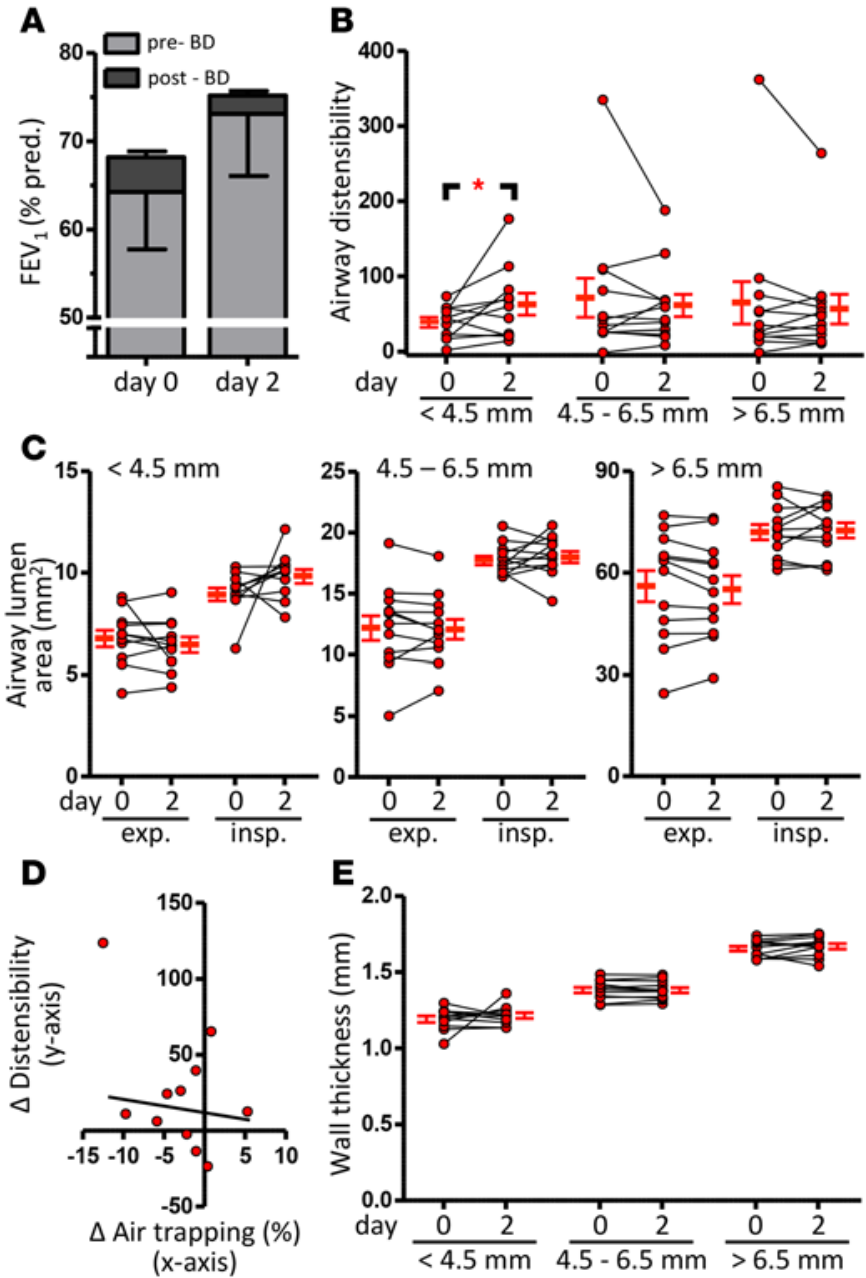

E

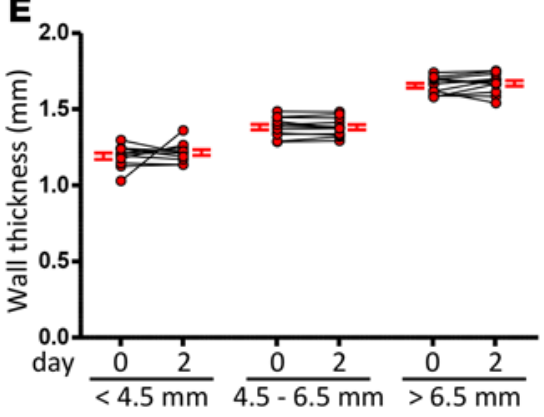

Figure 5. Airway effects of ivacaftor. (A) Forced expiratory volume in 1 second $\left(\mathrm{FEV}_{1}\right)$ (\% of pred.) on day 0 (before ivacaftor) and following 2 days of ivacaftor treatment (day 2). The light bar represents the average spirometric value prior to bronchodilator, and the dark bar represents the bronchodilator response. $n=12$ subjects. (B) Airway distensibility grouped by airway size. (C) Airway lumen area, grouped by airway size, from inspiratory (insp.) and expiratory (exp.) CT scans. (D) Lack of a correlation between change in distensibility of airways $<4.5 \mathrm{~mm}$ and change in air trapping after ivacaftor treatment. Each symbol represents a different subject. (E) Airway wall thickness was quantified from inspiratory CT scans on day $\mathbf{O}$ and day 2 . For panels $\mathbf{B}, \mathbf{C}$, and $\mathbf{E}$, airways were grouped by lumen diameter obtained from day 0 inspiratory scans. A total of 396 airways were analyzed (33 average airways/subject), ranging in size from 2.4-20.8 mm. Eighty-four airways were measured in the $<4.5 \mathrm{~mm}$ group (average of 7 airways/subject, $n=11$ subjects), 167 airways in the 4.5-6.5 mm group (average of 14 airways/subject, $n=12$ subjects), and 145 airways in the $>6.5 \mathrm{~mm}$ group (average of 12 airways/subject, $n=12$ subjects). In individual panels, each symbol represents a different subject, and connected symbols represent individual subjects before and after ivacaftor. Bars represent mean \pm SEM. ${ }^{*} P<0.05$ using a generalized linear mixed model to adjust for repeated measures on individual subjects. For D, the correlation measure ( $r$ ) was calculated using a linear mixed model to accommodate for a differing number of airway distensibility measurements for each subject.

We hypothesized that, compared with the bronchodilator response before ivacaftor, the bronchodilator response after ivacaftor would be reduced due to an ASM relaxant effect from CFTR restoration. On day 0 , the average bronchodilator response for $\mathrm{FEV}_{1}(\%$ predicted) was $3.9 \% \pm 0.7 \%$. After 2 days of ivacaftor treatment, this value significantly decreased to $2.1 \% \pm 0.5 \%(P=0.02$, paired $t$ test; Figure $5 \mathrm{~A})$. Because ivacaftor increased $\mathrm{FEV}_{1}$, the response to salbutamol may have been blunted due to a ceiling effect (Figure $5 \mathrm{~A}$ ), although there was no correlation between $\mathrm{FEV}_{1}$ after ivacaftor and the subsequent bronchodilator response. Nevertheless, a reduced bronchodilator response following CFTR restoration is consistent with ivacaftor's actions, at least in part, being mediated by a reduction in smooth muscle tone.

Airway distensibility. ASM regulates airway size (32), and increased smooth muscle tone decreases airway distensibility (33-35), the ability of an airway to distend with lung inflation. For the analysis, the measured airway segments were divided into 3 groups based on their starting lumen diameter $(<4.5 \mathrm{~mm}$, 4.5-6.5 mm, and $>6.5 \mathrm{~mm}$ ) obtained from baseline inspiratory CT scans. We only included airways identified in all 4 scans for a subject (days 0 and 2; inspiratory and expiratory scans). After 2 days of ivacaftor treatment, airway distensibility increased in airways $<4.5 \mathrm{~mm}$ (mean estimated difference, $14.39 \%$; $95 \%$ CI, 1.54-27.23; $P=0.03$ ) but not in airways $4.5-6.5 \mathrm{~mm}$ (mean estimated difference, $-1.33 \%$; $95 \% \mathrm{CI}$, $-12.85-10.20 ; P=0.82$ ) or in airways $>6.5 \mathrm{~mm}$ in diameter (mean estimated difference, $-8.9 \%$; $95 \% \mathrm{CI}$, $-33.91-16.11 ; P=0.48$ ) (Figure $5 \mathrm{~B}$ ). Following ivacaftor treatment, airways $<4.5 \mathrm{~mm}$ in diameter tended to decrease in absolute size on expiratory scans and increase in absolute size on inspiratory scans, but the differences were not statistically significant (for expiratory diameters $P=0.1$ and for inspiratory diameters $P=0.82 ;$ Figure 5C).

These data show that airway distensibility increased (in airways $<4.5 \mathrm{~mm}$ diameter) following CFTR restoration, a finding consistent with smooth muscle relaxation. However, these changes could be due to additional factors independent of ASM. We investigated 2 potential alternative mechanisms. A reduction 
A

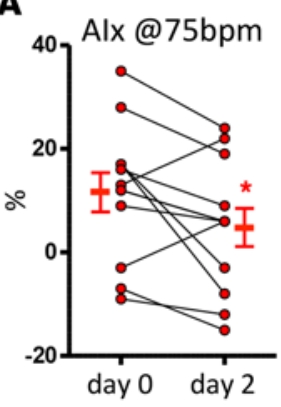

B

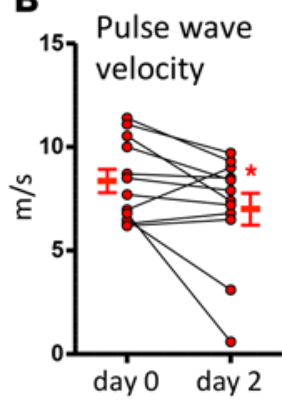

C

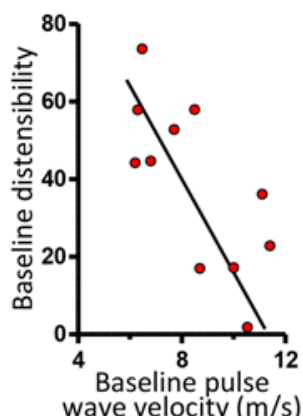

D

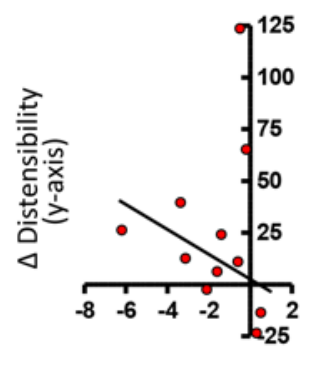

$\Delta$ Pulse wave velocity ( $\mathrm{m} / \mathrm{s}, \mathrm{x}$-axis)

Figure 6. Ivacaftor decreases vascular tone. Pulse wave analysis (PWA) was performed on day 0 (before ivacaftor) and following 2 days of ivacaftor treatment (day 2). (A) Heart rate-corrected (75 bpm) augmentation index (Alx@75). (B) Pulse wave velocity. (C) Correlation between baseline pulse wave velocity and baseline airway distensibility (airways $<4.5 \mathrm{~mm}$ diameter). (D) Correlation between change in pulse wave velocity and change in airway distensibility (airways $<4.5 \mathrm{~mm}$ diameter) after ivacaftor treatment. For individual panels, each symbol represents a different subject. In $\mathbf{A}$ and $\mathbf{B}$, connected symbols represent individual subjects before and after ivacaftor. Values are mean \pm SEM. A paired $t$ test $(\mathbf{A})$ or a Wilcoxon matched pairs signed-rank test (B) was performed. ${ }^{*} P<0.05$. For $\mathbf{C}$ and $\mathbf{D}$, the correlation measure $(r)$ was calculated using a linear mixed model to accommodate for a differing number of airway distensibility measurements for each subject.

in air trapping (as observed following ivacaftor treatment) could increase airway distensibility by allowing for periodic deep inspirations that have been shown to relax ASM (36). There was no correlation between changes in distensibility of airways $<4.5 \mathrm{~mm}$ and the reduction in air trapping $(P=0.36$; Figure $5 \mathrm{D})$. Lack of correlation might suggest that additional factors such as reduced mucus obstruction could also contribute to improvements in air trapping.

Changes in airway wall thickness could also influence airway distensibility independent of changes in smooth muscle tone $(37,38)$. CT-based airway measurements revealed that CFTR potentiation had no effect on wall thickness in airways $<4.5 \mathrm{~mm}$ (mean estimated difference, $0.0134 \mathrm{~mm}$; 95\% CI, -0.0230 0.0498; $P=0.47$ ), 4.5-6.5 mm (mean estimated difference, $-0.0028 \mathrm{~mm} ; 95 \% \mathrm{CI},-0.0237-0.0180 ; P=$ 0.79 ) or $>6.5 \mathrm{~mm}$ in diameter (mean estimated difference, $0.0147 \mathrm{~mm} ; 95 \% \mathrm{CI},-0.0574-0.0869 ; P=0.69$ ) (Figure 5E). Thus, increased airway distensibility is likely not related to ivacaftor-induced reductions in airway wall thickness.

Vascular smooth muscle tone. Based on our findings in the lung, we predicted that global restoration of CFTR function would decrease smooth muscle tone in a tissue outside the lung: the systemic vasculature. We chose to measure vascular smooth muscle tone for several reasons: (i) the readouts are noninvasive; (ii) vascular tone is less likely to be affected by airway infection and inflammation; and (iii) the measurement methods are independent of spirometry and CT scanning. Thus, finding an effect on vascular smooth muscle tone would support a smooth muscle cell-dependent response. To assess vascular smooth muscle tone (vascular stiffness), we measured the augmentation index and pulse wave velocity (39-41).

The augmentation index measures the degree to which the reflected systolic pulse wave augments systolic blood pressure. The pulse wave velocity is the propagation speed of the pressure pulse generated by systole from the heart to the periphery (42). Both the heart rate-corrected augmentation index (AIx@75) and pulse wave velocity should decrease if CFTR potentiation reduced vascular smooth muscle tone. Despite no significant changes in systolic blood pressure (120 $\pm 3 \mathrm{mmHg}$ vs. $117 \pm 2 \mathrm{mmHg}, P=0.3$ ), diastolic blood pressure ( $75 \pm 3 \mathrm{mmHg}$ vs. $71 \pm 2 \mathrm{mmHg}, P=0.1)$, or heart rate $(74 \pm 4 \mathrm{bpm}$ vs. $72 \pm 3 \mathrm{bpm}, P$ = 0.6), restoration of CFTR function reduced the AIx@75 $(P<0.05)$ and pulse wave velocity $(P=0.044$ for Wilcoxon matched pairs signed-rank test and $P=0.053$ for paired $t$ test) (Figure $6, \mathrm{~A}$ and B); these results were consistent with a reduction in vascular tone.

To further investigate an effect of CFTR restoration on smooth muscle function, we determined if ivacaftor-induced changes in airway distensibility correlated with changes in pulse wave velocity. First, we observed that baseline pulse wave velocity values were strongly correlated with baseline airway distensibility measures $(P<0.0001, \mathrm{r}=-0.54$; Figure $6 \mathrm{C})$. Second, we found that subjects with the greatest increase in distensibility of airways $<4.5 \mathrm{~mm}$ after CFTR restoration had the greatest reduction in pulse wave velocity $(P=0.03, \mathrm{r}=-0.24 ;$ Figure $6 \mathrm{D})$. Correlated changes in the airways and the vasculature are consistent with a common mechanism of action of CFTR restoration, leading to smooth muscle relaxation. 


\section{Discussion}

The goal of this study was to better understand the role of CFTR in smooth muscle function by using ivacaftor as a tool to restore CFTR activity. After only 2 days of ivacaftor treatment, significant improvements in CFTR function, airflow obstruction, airway distensibility, and vascular physiology were observed. The speed with which these physiological changes occurred, and the fact that changes were seen in the systemic vasculature, suggests that they were likely a primary consequence of CFTR restoration and are consistent with a relaxation effect of ivacaftor on smooth muscle.

Potential smooth muscle effect. CFTR is present in smooth muscle, and ASM abnormalities are observed in mice, pigs, and humans with CF $(4,7-9,14-16,43,44)$. Our findings shed new light on the effect of CFTR on smooth muscle function in people with CF.

First, these data further support a role for CFTR in smooth muscle physiology and suggest that: (i) some of the airflow obstruction in CF might be caused by abnormal ASM contraction and (ii) ivacaftor's rapid effects on pulmonary function might, at least in part, be due to bronchodilation via ASM relaxation. Interestingly, the effect of ivacaftor on the prebronchodilator $\mathrm{FEV}_{1}$ was greater than the effect of aerosolized salbutamol (albuterol) on $\mathrm{FEV}_{1}$ before ivacaftor treatment. Two potential explanations could account for this observation: (i) perhaps ivacaftor reaches airways that aerosolized salbutamol (albuterol) cannot, and their respective impact on $\mathrm{FEV}_{1}$ reflects that distribution, or (ii) perhaps ivacaftor affects a number of factors within the lung (e.g., smooth muscle, airway mucus, infection, etc.), and the pronounced response of $\mathrm{FEV}_{1}$ to ivacaftor reflects an amalgam of these factors. In contrast, an aerosolized bronchodilator's greatest acute effect is on ASM. Thus, the $\mathrm{FEV}_{1}$ gains in response to a bronchodilator might represent a fraction of ivacaftor's effects.

Second, the pulse wave analysis (PWA) studies are consistent with a smooth muscle-specific effect. Because PWA investigates an environment outside of the lung, it enables assessment of smooth muscle in a manner experimentally isolated from the lung and, therefore, largely independent of changes in factors such as mucus. The significant correlation between change in pulse wave velocity and change in airway distensibility is suggestive of a relaxation effect of ivacaftor on vascular and airway smooth muscle.

Finally, in the current study, the effects on airway distensibility were only found in the smaller-sized airways. Similarly, Kelly et al. (45) found in asthmatics that, after bronchodilator treatment, airway compliance or distensibility increased more in smaller-sized airways. It is likely that the relative distribution of smooth muscle throughout the airway tree plays a role in this size dependency. In absolute terms, the amount of ASM decreases as airway size decreases for healthy controls. However, when normalized to airway size, ASM mass increases with decreasing airway size $(46,47)$. Perhaps the effect on airway distensibility in our study was only observed in airways $<4.5 \mathrm{~mm}$ due to smooth muscle distribution. In addition, the smooth muscle distribution could be altered by chronic disease (47).

Other potential mechanisms. While our data suggest that the improvements in airflow obstruction could be due to a direct effect on ASM function, other potential mechanisms are possible. First, it is possible the smooth muscle effects could be due to a reduction in airway inflammation. For example, a separate study of our patient cohort found that ivacaftor treatment decreased the expression of interferon $\gamma$ inducible proteins on blood monocytes (48). However, the landmark GOAL study, which studied patients before and after ivacaftor, showed no significant changes in sputum markers of inflammation (24). Furthermore, it is unknown whether ASM exposed to years or decades of chronic inflammation would acutely respond to reduced inflammation, even if clinically meaningful changes occurred within the 48-hour time frame studied here.

Second, a component of ivacaftor's beneficial effects could be linked to enhanced airway mucus clearance. This hypothesis is supported by recent data demonstrating improvements in mucociliary clearance after one month of ivacaftor treatment in people with CF and the G551D-CFTR mutation (24). In the current study, we did not quantify mucociliary clearance or mucus biophysical properties before and after ivacaftor. Thus, whether ivacaftor has an acute effect on these parameters remains unknown. However, changes in mucociliary clearance are unlikely to account for the observed effects of CFTR potentiation on airway distensibility or vascular tone.

Strengths and limitations. Strengths of this study include the following: (i) The short study duration limited the contribution of secondary factors, enabling investigation of effects likely primary to CFTR potentiation. (ii) Assessment of vascular smooth muscle function (using PWA) provided additional support for a smooth muscle-specific effect that is likely independent of secondary factors in the lung environment. (iii) Our findings are supported by studies in both animals and humans. In ex vivo and in vivo 
animal experiments, increased bronchomotor tone decreases airway distensibility (49-52). Moreover, bronchial thermoplasty, an asthma treatment that likely affects ASM, diminishes methacholine-induced reductions in airway distensibility $(35,53)$. Most studies in human asthmatics show that airway distensibility is reduced (54-60). However, in asthmatics, the contribution of bronchomotor tone to reductions in airway distensibility is less clear, and significant differences between studies in asthma severity, bronchodilator responsiveness, study design, and methods for determining airway distensibility likely account for the differing findings $(45,54,57)$. (iv) Prospective, standardized, whole-lung, inspiratory and expiratory CT scans permitted detailed measurements of lungs and spatial matching of the airways on an individual basis for before-after comparisons. (v) Subjects served as their own control (before and after ivacaftor), thereby reducing confounding factors.

Limitations include the following: (i) We used an abbreviated study duration of 2 days to limit secondary effects of CFTR potentiation. Whether similar effects on airway distensibility and PWA will be observed at later time points remains to be determined. (ii) We did not investigate other proposed mechanisms of CF lung disease, including changes in airway surface liquid $\mathrm{pH}$, mucus properties, or mucociliary clearance that might have contributed to improved lung function after ivacaftor. (iii) Our spirometry, airway distensibility data, and vascular studies support a smooth muscle-dependent effect. However, we are not able to exclude that some of our observed findings could be related to CFTR restoration in other cell types, such as epithelial cells or neurons (61), although our earlier studies in newborn CF pigs support a direct effect of CFTR potentiation on smooth muscle (16). (iv) We cannot exclude the possibility that an off-target effect of ivacaftor affected smooth muscle. However, studies in humans suggest that if there are off-target effects of ivacaftor, they may not affect smooth muscle function since ivacaftor did not improve airflow in people with CF homozygous for the F508-del CFTR mutation (62). Also arguing against an off-target effect, ivacaftor reduced airway narrowing in porcine $C F T R^{+/+}$airways but not porcine $C F T R^{-/-}$airways. Furthermore, no effect on airway narrowing was seen in murine $C F T R^{+/+}$airways, presumably because ivacaftor lacks activity on murine CFTR and because off-target effects were absent (16). (v) Our study had a small sample size; however, we were still able to detect differences.

Implications. These data have a number of important implications. First, this study demonstrates that CFTR corrector/potentiator studies, with limited participant numbers and duration, can be very informative when studying a very effective compound. Despite limited sample size and study duration, we found a marked physiological response. Second, the improvement in lung function apparent within 48 hours, as measured by $\mathrm{FEV}_{1}$, nearly equaled the improvements in lung function observed after months of ivacaftor therapy $(19,22,24,63-66)$. This implies that a major component of ivacaftor's mechanism is fast acting. Finally, ivacaftor increased airway distensibility and decreased vascular tone. These findings suggest that ivacaftor may relax smooth muscle and provide a link between CFTR function and smooth muscle tone. Thus, the abnormal smooth muscle function observed in animal models and people with CF may be, in part, a primary consequence of CFTR disruption, and abnormal smooth muscle function may contribute to the pathogenesis of CF lung disease.

\section{Methods}

Subjects and study design. We conducted a prospective, observational study at a single CF center (National Referral Centre for Adult Cystic Fibrosis, Dublin, Ireland) of adult subjects with CF who had at least 1 G551D-CFTR allele. Other limited studies on monocyte function from this patient cohort have been reported, and in that report, we provided values of sweat chloride and $\mathrm{FEV}_{1}$ on day 0 that are also shown here (Figure 1, A and B, and ref. 48). Research participants were studied on day 0 (visit 1, prior to starting ivacaftor treatment) and again on day 2 (visit 2, approximately 48 hours after starting ivacaftor). Inclusion criteria included ages $\geq 18$ years (both male and female subjects were enrolled), a clinical diagnosis of $\mathrm{CF}$ and the G551D-CFTR mutation on at least 1 allele, clinical stability with no significant changes in health status within 14 days prior to visit 1 , and a negative serum pregnancy test at screening for females of childbearing potential. Exclusion criteria included participation in the VX-770 Extended Access Program or use of ivacaftor within 6 months prior to visit 1; an acute upper or lower respiratory infection, pulmonary exacerbation, or changes in therapy (including antibiotics) for pulmonary disease within 4 weeks of visit 1 ; history of solid organ transplantation; pregnant or breastfeeding; ongoing participation in another therapeutic clinical study or prior participation in an investigational drug study within 30 days before screening; use of any inhibitors or inducers of cytochrome P450 3A4; any non-CF-related illness within 2 weeks before visit 
1; or abnormal renal or liver function, at screening. Standard therapy for CF was allowed to continue except for inhaled bronchodilators that were held the morning of visits.

On visit 1 (day 0 - prior to beginning ivacaftor), subject's height, weight, blood pressure, heart rate, and respiratory rate were recorded. Spirometry, chest CT scanning, sweat chloride measurements, and PWA were performed. Following pretreatment study assessments, subjects began standard ivacaftor treatment (150 mg orally every 12 hours; Vertex Pharmaceuticals). Subjects returned approximately 48 hours later (visit 2) and repeated the assessments performed on visit 1 . Study adherence was $100 \%$.

Sweat chloride. Sweat was collected with the Macroduct collection system (Wescor), and sweat chloride levels were measured using routine laboratory techniques.

Chest CT. Subjects were positioned supine within a multidector-row CT scanner (GE HD750: GE Healthcare). Full lung scans were acquired during breath holds at coached TLC (inspiratory scan) and coached RV (expiratory scan) on visit 1 and visit 2 . The scanning protocol was based on the SPIROMICS study protocol (67-70). The scan parameters for the GE HD750 were as follows: $120 \mathrm{kV}$ and current of $180 \mathrm{~mA}$ for inspiratory scans and $100 \mathrm{~mA}$ for expiratory scans. The slice thickness was $0.625 \mathrm{~mm}$ with an interval of $0.5 \mathrm{~mm}$ and a pitch of $0.984: 1$. Spatial resolution was matched at baseline and follow-up using a similar diameter field of view for both scanning sessions. Scans were reconstructed with filtered back projection and standard kernel, and they were deidentified prior to transfer to the University of Iowa for analysis. Three deep breaths to standardize lung volume history preceded scans. Subjects were coached to reach TLC and RV, and the maneuvers were rehearsed prior to scanning.

CT scan analysis. Quantitative CT analysis was completed utilizing the Apollo Software (VIDA Diagnostics Inc.). The lungs and lung lobes were automatically segmented. Similar to other studies, we defined image-based air trapping as the percentage of lung CT scan voxels less than -856 HU at RV (71). Apollo also semiautomatically segmented the airway tree centerline from which airway tree geometry (including luminal area, luminal diameter, and airway wall thickness) is automatically generated for an orientation perpendicular to the centerline. Through the automated labeling of the airway segments (72), airways were spatially matched on an individual basis for before-after comparisons. Measurements were sought for 42 individual airway segments per scan, although results are only reported for individual airway segments that were successfully identified in all 4 scans from an individual subject (day 0 and day 2; inspiratory and expiratory scans). These 42 airways included both segmental and subsegmental airway branches. Segmentation started with the trachea through RB10 and LB10 with the additional 2 airway generations respectively beyond RB1 (e.g., RB1a, RB1ai), RB4, RB10, LB1, and LB10, constituting one airway path for each of the 5 lung lobes, similar to Smith et al. (73).

Airway distensibility. The airway measurements obtained from the CT scans were used to generate an estimate of airway distensibility. Airway distensibility was calculated on a per-airway basis as follows: ([inspiratory airway lumen area - expiratory airway lumen area]/expiratory airway lumen area) $\times 100$ (35) Only airways with both a before and after ivacaftor distensibility measure are reported.

Spirometry. Spirometric measurements were obtained in accordance with the American Thoracic Society Standards (74). Spirometry was performed before and after inhaled bronchodilator therapy (Ventolin [salbutamol] 2 puffs - $100 \mathrm{mcg}$ ).

$P W A$. PWA measurements were obtained for assessment of central blood pressure and arterial tone using a SphygmorCor machine (Core Medical), per the manufacturer's instructions. Measurements were obtained from the carotid and radial arteries from supine positioned subjects. Pulse wave velocity was calculated through division of transit distance by time. AIx measurements were corrected for a heart rate of 75 beats per minute (AIx@75). To demonstrate that these measurements were reproducible and did not vary without treatment, we also collected pulse wave profiles of people with CF (of any mutation type) who were not subject to changes in treatment at baseline and 2 days later. At baseline, the average AIx@75 was 11.8\% $\pm 2.8 \%$ and, after 2 days (with no treatment or intervention), was $11.9 \% \pm 3.9 \%(P>0.05, n=13$ subjects).

Statistics. For distensibility, wall thickness, expiratory lumen area, and inspiratory lumen area, the mean for each sized airway group was modeled at day 0 and day 2 using a generalized linear mixed model to adjust for repeated measures on subjects. To accommodate the right-skewed nature of the measures, a $\gamma$ distribution was specified. Means for each sized airway group were modeled separately due to heterogeneous variances. The estimate of the mean difference between day 2 and day 0 was examined for significance. The association between changes in distensibility (day 2 to day 0 ) and corresponding changes in other measured values was characterized using a linear mixed model to accommodate the repeated measures on subjects. 
Although the original measures exhibit a right-skewed distribution, the distribution of the differences is reasonably symmetric, allowing for the assumption of approximate normality. The test for the estimate of the slope was assessed to determine if the trend was significantly different from 0 , and a correlation measure based on a linear mixed model was calculated. For most of the data with a single value per subject per time point, we used the 2-tailed, paired $t$ test based on the principle that it is the most powerful inferential procedure under normality. We analyzed the data for normality using Q-Q plots. In general, the normality assumption was supported by the Q-Q plots. However, if the Q-Q plot suggested deviations from normality, we confirmed the result of the paired $t$ test with the Wilcoxon matched pairs signed-rank test and, in those cases, report $P$ values from both statistical analyses. Pearson's correlation coefficient (r) was used to assess the strength of linear relationships between 2 variables. All tests used a significance level of 0.05 . Analyses were performed in either SAS 9.4 (SAS Institute) or Prism (GraphPad).

Study approval. The Research Ethics Committee of St. Vincent's University Hospital approved this study, and written informed consent was obtained from participants prior to inclusion in the study.

\section{Author contributions}

RJA, KBH, PKS, MJW, EFM, and DAS conceptualized and designed the research. RJA, KBH, JDD, BG, JLL, CGG, PKS, MJW, EFM, and DAS performed studies and data acquisition. RJA, KBH, JDD, JKB, JPS, TJG, AJF, JEC, EAH, PKS, MJW, EFM, and DAS analyzed data. RJA, KBH, JPS, TJG, AJF, EAH, PKS, MJW, EFM, and DAS interpreted results of experiments. RJA, JKB, JEC, PKS, MJW, EFM, and DAS prepared figures. RJA, PKS, MJW, EFM, and DAS drafted the manuscript. RJA, KBH, JDD, BG, JLL, JKB, CGG, JPS, TJG, AJF, JEC, EAH, PKS, MJW, EFM, and DAS edited, reviewed, and approved final the version of the manuscript.

\section{Acknowledgments}

We thank all of the study participants and the study coordinators. This work was funded in part, including costs for CT scans, from an unrestricted grant from the Vertex Investigator-Initiated Studies Program, the Cystic Fibrosis Foundation Research and Development Program (University of Iowa and University of Washington), and the NIH (P01 HL091842 and T32 HL007638). An Early Excellence Award from the American Asthma Foundation supported D.A. Stoltz. K. Hisert was supported by the Cystic Fibrosis Foundation LeRoy Matthews Physician Scientist award. P.K. Singh was supported by grants from NIH (R01 HL110879, R01 AI101307, and K24 HL102246), European Commission (Seventh Framework Programme Project 603038 CFMatters), and the Burroughs Wellcome Fund. We thank T. Mayhew for excellent assistance. We also thank M. Abou Alaiwa, D. Cook, L. Durairaj, M. Eberlein, P. McCray, J. Newell, J. Wilson, and J. Zabner for insightful discussions and guidance.

Address correspondence to: David A. Stoltz, Department of Internal Medicine, Pappajohn Biomedical Institute, University of Iowa, 169 Newton Rd, 6322 PBDB, Iowa City, Iowa 52242, USA. Phone: 319.356.4419; E-mail: david-stoltz@uiowa.edu. Or to: Edward F. McKone, National Referral Centre for Adult Cystic Fibrosis, St. Vincent's University Hospital and University College Dublin School of Medicine, Dublin, Ireland. Phone: 353.1.2213611; E-mail: e.mckone@svuh.ie.

1. Stoltz DA, Meyerholz DK, Welsh MJ. Origins of cystic fibrosis lung disease. N Engl J Med. 2015;372(4):351-362.

2. Fisher JT, Zhang Y, Engelhardt JF. Comparative biology of cystic fibrosis animal models. Methods Mol Biol. 2011;742:311-334

3. Rogan MP, et al. Pigs and humans with cystic fibrosis have reduced insulin-like growth factor 1 (IGF1) levels at birth. Proc Natl Acad Sci U S A. 2010;107(47):20571-20575.

4. Meyerholz DK, et al. Loss of cystic fibrosis transmembrane conductance regulator function produces abnormalities in tracheal development in neonatal pigs and young children. Am J Respir Crit Care Med. 2010;182(10):1251-1261.

5. Abou Alaiwa MH, et al. Neonates with cystic fibrosis have a reduced nasal liquid pH; a small pilot study. J Cyst Fibros. 2014;13(4):373-377.

6. Fischer AJ, et al. Tracheomalacia is associated with lower FEV1 and Pseudomonas acquisition in children with CF. Pediatr Pulmonol. 2014;49(10):960-970.

7. Michoud MC, et al. Role of the cystic fibrosis transmembrane conductance channel in human airway smooth muscle. Am $J$ Respir Cell Mol Biol. 2009;40(2):217-222.

8. Regamey N, et al. Increased airway smooth muscle mass in children with asthma, cystic fibrosis, and non-cystic fibrosis bronchiectasis. Am J Respir Crit Care Med. 2008;177(8):837-843.

9. Hays SR, Ferrando RE, Carter R, Wong HH, Woodruff PG. Structural changes to airway smooth muscle in cystic fibrosis. Tho- 
rax. 2005;60(3):226-228.

10. Balfour-Lynn IM, Elborn JS. "CF asthma": what is it and what do we do about it? Thorax. 2002;57(8):742-748.

11. Sanchez I, Powell RE, Pasterkamp H. Wheezing and airflow obstruction during methacholine challenge in children with cystic fibrosis and in normal children. Am Rev Respir Dis. 1993;147(3):705-709.

12. Hiatt P, Eigen H, Yu P, Tepper RS. Bronchodilator responsiveness in infants and young children with cystic fibrosis. Am Rev Respir Dis. 1988;137(1):119-122.

13. Kent BD, Lane SJ, van Beek EJ, Dodd JD, Costello RW, Tiddens HA. Asthma and cystic fibrosis: a tangled web. Pediatr Pulmonol. 2014;49(3):205-213.

14. Vandebrouck C, et al. Evidence that CFTR is expressed in rat tracheal smooth muscle cells and contributes to bronchodilation. Respir Res. 2006;7:113.

15. Norez C, Jayle C, Becq F, Vandebrouck C. Bronchorelaxation of the human bronchi by CFTR activators. Pulm Pharmacol Ther. 2014;27(1):38-43.

16. Cook DP, et al. Cystic Fibrosis Transmembrane Conductance Regulator in Sarcoplasmic Reticulum of Airway Smooth Muscle. Implications for Airway Contractility. Am J Respir Crit Care Med. 2016;193(4):417-426.

17. Adam RJ, et al. Air trapping and airflow obstruction in newborn cystic fibrosis piglets. Am J Respir Crit Care Med. 2013;188(12):1434-1441.

18. Accurso FJ, et al. Effect of VX-770 in persons with cystic fibrosis and the G551D-CFTR mutation. N Engl J Med. 2010;363(21):1991-2003.

19. Ramsey BW, et al. A CFTR potentiator in patients with cystic fibrosis and the G551D mutation. N Engl J Med. 2011;365(18):1663-1672.

20. Yu H, et al. Ivacaftor potentiation of multiple CFTR channels with gating mutations. J Cyst Fibros. 2012;11(3):237-245.

21. Van Goor F, et al. Rescue of CF airway epithelial cell function in vitro by a CFTR potentiator, VX-770. Proc Natl Acad Sci U S A. 2009;106(44):18825-18830.

22. Davies JC, et al. Efficacy and safety of ivacaftor in patients aged 6 to 11 years with cystic fibrosis with a G551D mutation. Am J Respir Crit Care Med. 2013;187(11):1219-1225.

23. McKone EF, et al. Long-term safety and efficacy of ivacaftor in patients with cystic fibrosis who have the Gly551Asp-CFTR mutation: a phase 3, open-label extension study (PERSIST). Lancet Respir Med. 2014;2(11):902-910.

24. Rowe SM, et al. Clinical mechanism of the cystic fibrosis transmembrane conductance regulator potentiator ivacaftor in G551D-mediated cystic fibrosis. Am J Respir Crit Care Med. 2014;190(2):175-184.

25. Sawicki GS, et al. Sustained benefit from ivacaftor demonstrated by combining clinical trial and cystic fibrosis patient registry data. Am J Respir Crit Care Med. 2015;192(7):836-842.

26. Ivacaftor (Kalydeco) [prescribing information]. Cambridge, Massachusetts, USA: Vertex Pharmaceuticals; 2012.

27. Hall GL, et al. Air trapping on chest CT is associated with worse ventilation distribution in infants with cystic fibrosis diagnosed following newborn screening. PLoS One. 2011;6(8):e23932.

28. Sly PD, et al. Lung disease at diagnosis in infants with cystic fibrosis detected by newborn screening. Am J Respir Crit Care Med 2009;180(2):146-152.

29. Brody AS, Klein JS, Molina PL, Quan J, Bean JA, Wilmott RW. High-resolution computed tomography in young patients with cystic fibrosis: distribution of abnormalities and correlation with pulmonary function tests. J Pediatr. 2004;145(1):32-38

30. Sly PD, et al. Risk factors for bronchiectasis in children with cystic fibrosis. N Engl J Med. 2013;368(21):1963-1970.

31. Bonnel AS, et al. Quantitative air-trapping analysis in children with mild cystic fibrosis lung disease. Pediatr Pulmonol. 2004;38(5):396-405

32. Gerthoffer WT. Regulation of the contractile element of airway smooth muscle. Am J Physiol. 1991;261(2 pt 1):L15-28.

33. Olsen CR, Stevens AE, McIlroy MB. Rigidity of tracheae and bronchi during muscular constriction. J Appl Physiol. 1967;23(1):27-34

34. Jensen A, Atileh H, Suki B, Ingenito EP, Lutchen KR. Selected contribution: airway caliber in healthy and asthmatic subjects: effects of bronchial challenge and deep inspirations. J Appl Physiol (1985). 2001;91(1):506-515.

35. Brown RH, Wizeman W, Danek C, Mitzner W. Effect of bronchial thermoplasty on airway distensibility. Eur Respir J. $2005 ; 26(2): 277-282$.

36. West AR, Needi ET, Mitchell HW, McFawn PK, Noble PB. Airways dilate to simulated inspiratory but not expiratory manoeuvres. Eur Respir J. 2012;40(2):455-461.

37. Ward C, et al. Reduced airway distensibility, fixed airflow limitation, and airway wall remodeling in asthma. Am J Respir Crit Care Med. 2001;164(9):1718-1721.

38. McParland BE, Macklem PT, Pare PD. Airway wall remodeling: friend or foe? J Appl Physiol (1985). 2003;95(1):426-434

39. Dobrin PB. Mechanical properties of arterises. Physiol Rev. 1978;58(2):397-460.

40. Sutton-Tyrrell K, et al. Elevated aortic pulse wave velocity, a marker of arterial stiffness, predicts cardiovascular events in wellfunctioning older adults. Circulation. 2005;111(25):3384-3390.

41. Kelly RP, Millasseau SC, Ritter JM, Chowienczyk PJ. Vasoactive drugs influence aortic augmentation index independently of pulse-wave velocity in healthy men. Hypertension. 2001;37(6):1429-1433.

42. Nichols WW. Clinical measurement of arterial stiffness obtained from noninvasive pressure waveforms. Am J Hypertens. 2005; 18(1 pt 2):3S-10S.

43. Wallace HL, Southern KW, Connell MG, Wray S, Burdyga T. Abnormal tracheal smooth muscle function in the CF mouse Physiol Rep. 2013;1(6):e00138.

44. Wilke M, et al. Mouse models of cystic fibrosis: phenotypic analysis and research applications. J Cyst Fibros. 2011;10(suppl 2):S152-S171.

45. Kelly VJ, Brown NJ, King GG, Thompson BR. The bronchodilator response of in vivo specific airway compliance in adults with asthma. Ann Biomed Eng. 2011;39(3):1125-1135

46. Matsuba K, Thurlbeck WM. A morphometric study of bronchial and bronchiolar walls in children. Am Rev Respir Dis. 1972;105(6):908-913. 
47. James A, Carroll N. Airway smooth muscle in health and disease; methods of measurement and relation to function. Eur Respir J. 2000;15(4):782-789.

48. Hisert $\mathrm{KB}$, et al. Ivacaftor-induced proteomic changes suggest monocyte defects may contribute to the pathogenesis of cystic fibrosis. Am J Respir Cell Mol Biol. 2016;54(4):594-507.

49. Hahn HL, Watson A, Wilson AG, Pride NB. Influence of bronchomotor tone on airway dimensions and resistance in excised dog lungs. J Appl Physiol Respir Environ Exerc Physiol. 1980;49(2):270-278.

50. Gunst SJ, Stropp JQ, Service J. Mechanical modulation of pressure-volume characteristics of contracted canine airways in vitro. J Appl Physiol (1985). 1990;68(5):2223-2229.

51. Brown RH, Mitzner W. Effect of lung inflation and airway muscle tone on airway diameter in vivo. J Appl Physiol (1985). 1996;80(5):1581-1588.

52. Brown RH, Mitzner W, Bulut Y, Wagner EM. Effect of lung inflation in vivo on airways with smooth muscle tone or edema. J Appl Physiol (1985). 1997;82(2):491-499.

53. Brown RH, Wizeman W, Danek C, Mitzner W. In vivo evaluation of the effectiveness of bronchial thermoplasty with computed tomography. J Appl Physiol (1985). 2005;98(5):1603-1606.

54. Brown NJ, Salome CM, Berend N, Thorpe CW, King GG. Airway distensibility in adults with asthma and healthy adults, measured by forced oscillation technique. Am J Respir Crit Care Med. 2007;176(2):129-137.

55. Kermode JA, et al. The effect of airway remodelling on airway hyper-responsiveness in asthma. Respir Med. 2011;105(12):1798-1804.

56. Pyrgos G, Scichilone N, Togias A, Brown RH. Bronchodilation response to deep inspirations in asthma is dependent on airway distensibility and air trapping. J Appl Physiol (1985). 2011;110(2):472-479.

57. Kelly VJ, Brown NJ, Sands SA, Borg BM, King GG, Thompson BR. Effect of airway smooth muscle tone on airway distensibility measured by the forced oscillation technique in adults with asthma. J Appl Physiol (1985). 2012;112(9):1494-1503.

58. Brown RH, Scichilone N, Mudge B, Diemer FB, Permutt S, Togias A. High-resolution computed tomographic evaluation of airway distensibility and the effects of lung inflation on airway caliber in healthy subjects and individuals with asthma. Am J Respir Crit Care Med. 2001;163(4):994-1001

59. Johns DP, Wilson J, Harding R, Walters EH. Airway distensibility in healthy and asthmatic subjects: effect of lung volume history. J Appl Physiol (1985). 2000;88(4):1413-1420.

60. Brown RH, Mitzner W, Wagner E, Permutt S, Togias A. Airway distension with lung inflation measured by HRCT. Acad Radiol. 2003;10(10):1097-1103.

61. Reznikov LR, et al. CFTR-deficient pigs display peripheral nervous system defects at birth. Proc Natl Acad Sci U S A. 2013;110(8):3083-3088.

62. Flume PA, et al. Ivacaftor in subjects with cystic fibrosis who are homozygous for the F508del-CFTR mutation. Chest. 2012;142(3):718-724.

63. Polenakovik HM, Sanville B. The use of ivacaftor in an adult with severe lung disease due to cystic fibrosis ( $\Delta$ F508/G551D). J Cyst Fibros. 2013;12(5):530-531.

64. Harrison MJ, Murphy DM, Plant BJ. Ivacaftor in a G551D homozygote with cystic fibrosis. N Engl J Med. 2013;369(13):1280-1282.

65. Barry PJ, et al. Effects of ivacaftor in patients with cystic fibrosis who carry the G551D mutation and have severe lung disease. Chest. 2014;146(1):152-158.

66. Hoare S, et al. Ivacaftor imaging response in cystic fibrosis. Am J Respir Crit Care Med. 2014;189(4):484.

67. Yamashiro T, et al. Collapsibility of lung volume by paired inspiratory and expiratory CT scans: correlations with lung function and mean lung density. Acad Radiol. 2010;17(4):489-495.

68. Zaporozhan J, et al. Paired inspiratory/expiratory volumetric thin-slice CT scan for emphysema analysis: comparison of different quantitative evaluations and pulmonary function test. Chest. 2005;128(5):3212-3220.

69. Couper D, et al. Design of the Subpopulations and Intermediate Outcomes in COPD Study (SPIROMICS). Thorax 2014;69(5):491-494.

70. Newell JD Jr, Sieren J, Hoffman EA. Development of quantitative computed tomography lung protocols. J Thorac Imaging 2013;28(5):266-271.

71. Busacker A, et al. A multivariate analysis of risk factors for the air-trapping asthmatic phenotype as measured by quantitative CT analysis. Chest. 2009;135(1):48-56.

72. Tschirren J, McLennan G, Palagyi K, Hoffman EA, Sonka M. Matching and anatomical labeling of human airway tree. IEEE Trans Med Imaging. 2005;24(12):1540-1547.

73. Smith BM, et al. Comparison of spatially matched airways reveals thinner airway walls in COPD. The Multi-Ethnic Study of Atherosclerosis (MESA) COPD Study and the Subpopulations and Intermediate Outcomes in COPD Study (SPIROMICS) Thorax. 2014;69(11):987-996.

74. Standardization of Spirometry, 1994 Update. American Thoracic Society. Am J Respir Crit Care Med. 1995;152(3):1107-1136 